\title{
2,4,6-tris[bis(1H-tetrazol-5-yl)amino]-1,3,5-triazine as a nitrogen-rich material
}

\author{
MUDDAMARRI HANUMANTHA RAO ${ }^{\mathrm{a}}$, VIKAS D GHULE $^{\mathrm{b}}$ and \\ KRISHNAMURTHI MURALIDHARAN ${ }^{\mathrm{a}, \mathrm{c}, *}$ (D) \\ ${ }^{a}$ Advanced Centre of Research in High Energy Materials (ACRHEM), University of Hyderabad, Hyderabad, \\ Telengana 500 046, India \\ ${ }^{b}$ Department of Chemistry, National Institute of Technology, Kurukshetra, Haryana 136 119, India \\ ${ }^{\mathrm{c}}$ School of Chemistry, University of Hyderabad, Hyderabad, Telengana 500 046, India \\ E-mail:kmsc@uohyd.ernet.in
}

MS received 14 March 2017; revised 21 April 2017; accepted 21 April 2017

\begin{abstract}
This paper describes the synthesis, characterization and energetic properties of tetrazole and triazine-based thermally stable, insensitive nitrogen-rich high energy density materials of N, N', N"-(1,3,5triazine-2,4,6-triyl) tris(N-cyanocyanamide) [1] and 2,4,6-tris[bis(1H-tetrazol-5-yl)amino]-1,3,5-triazine [2]. The compound $\mathbf{2}$ is the first example for compounds having six tetrazole units on a triazine ring.
\end{abstract}

Keywords. Nitrogen rich compounds; high energy materials; density functional theory.

\section{Introduction}

Synthesizing more potent energetic compounds is an exciting task for the synthetic chemists due to their unknown hazardous nature. Besides, combining high positive heat of formation (HOF) and better density in the same molecule to achieve high detonation performance with the straightforward synthetic route is challenging. Hence, research on developing new strategies and methodologies to synthesize nitrogen-rich energetic compounds are getting tremendous importance due to their numerous applications in gas generators, propellants, and explosives. ${ }^{1-5}$ Nitrogen-rich compounds help to achieve high densities, high HOFs and stability due to nitrogen content, $\mathrm{C}-\mathrm{N}$ and $\mathrm{N}-\mathrm{N}$ bonds, and aromaticity. On decomposition, the nitrogen-rich molecules would produce a large quantity of eco-friendly nitrogen gas and large amounts of energy. ${ }^{6-8}$ The low molecular weight nitrogen compounds are known to give more thrust to a rocket motor because of their high kinetic energy.

Designing molecules with more number of tetrazole groups is an attractive strategy to produce nitrogen-rich molecules. Our group reported ${ }^{9}$ several tetra anionic nitrogen rich tetrazole-based energetic salts synthesized from simple starting materials in good yields. In this context, we have synthesized nitrogen-rich containing

\footnotetext{
*For correspondence
}

triazine and tetrazole rings based on reference methods. ${ }^{10-13}$ Herein, we report the synthesis of nitrogen-rich molecules, N, N', N'-(1,3,5-triazine-2,4,6-triyl) tris $(\mathrm{N}$ cyanocyanamide) (1) and 2,4,6-tris[bis(1H-tetrazol-5yl)amino]-1,3,5-triazine (2) (Figure 1). Two newly synthesized molecules $\mathbf{1}$ and $\mathbf{2}$ were thermally stable up to $300^{\circ} \mathrm{C}$, not sensitive to impact and friction, and possess higher density and HOF. To the best of our knowledge, the compound $\mathbf{2}$ is the first example of a compound having six tetrazole moieties in a single molecule constructed on triazine ring.

\section{Experimental}

Caution: The compounds $\mathbf{1}$ and $\mathbf{2}$ were not sensitive to moisture and stable until ignited inside the bomb calorimeter. However, the molecules with high nitrogen content and energy are expected to be sensitive towards shock, friction and heat. Hence, appropriate safety precautions, such as kevlar gloves, face shield, leather jacket, earplugs, Teflon spatulas, safety goggles and earthened equipment must be employed.

\subsection{Materials and Instruments}

Cyanuric Chloride (Sigma-Aldrich), Sodium dicyanamide (Sigma-Aldrich), Sodium Azide (Merck), Zinc bromide (Alfa-Aesar) were commercially available. IR spectra were recorded on JASCO 5300 FTIR with $\mathrm{KBr}$ pellet. ${ }^{1} \mathrm{H}$ and ${ }^{13} \mathrm{C}$ NMR were recorded on Bruker Avance $500 \mathrm{MHz}$ in $5 \mathrm{~mm}$ NMR tubes at room temperature. The isotropic solid state 
<smiles>N#CN(C#N)c1nc(N(C#N)C#N)nc(N(C#N)C#N)n1</smiles>

[1]

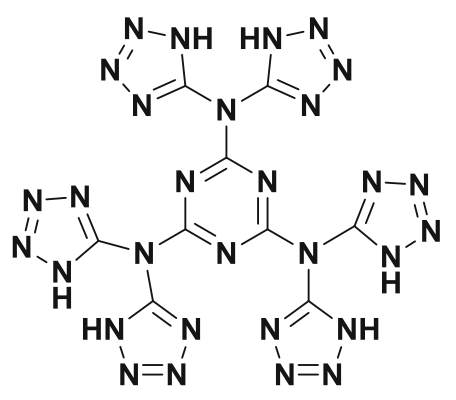

[2]
Figure 1. Molecular structures of compounds $\mathbf{1}$ and 2.

spectra were recorded by MAS speed of $4 \mathrm{kHz}$ in a zirconia rotor using Bruker Avance 400 spectrometer. Chemical shifts were recorded based on tetramethylsilane as reference. LCMS was recorded on 2010A, Shimadzu, Japan. The HRMS were recorded on a Bruker Maxis instrument. Elemental analysis was recorded on EA1112. Thermal decomposition experiments were performed under UHP nitrogen atmosphere in a TA instrument (SDT Q600). The samples $(\sim 1 \mathrm{mg})$ were analysed from room temperature to $800^{\circ} \mathrm{C}$ with a heating rate $10^{\circ} \mathrm{C} / \mathrm{min}$.

\subsection{Synthesis of $N, N^{\prime}, N^{\prime \prime}-(1,3,5$-triazine-2,4,6- triyl)tris( $N$-cyanocyanamide (1)}

An aqueous solution of sodium dicyanamide $(0.80 \mathrm{~g}, 9$ $\mathrm{mmol}$ ) (in $15 \mathrm{~mL}$ of deionized water) was added dropwise to the slurry of cyanuric chloride $(0.18 \mathrm{~g}, 1 \mathrm{mmol})$ in acetone $(50 \mathrm{~mL})$ at $55^{\circ} \mathrm{C}$. The reaction mixture was stirred continuously for $2 \mathrm{~h}$. After cooling to room temperature, the reaction mixture was cooled by the addition of crushed ice and continued the stirring for $30 \mathrm{~min}$. Then, the mixture was filtered and washed with water to obtain a white solid product of $\mathrm{N}, \mathrm{N}$ ', $\mathrm{N}$ '-(1,3,5-triazine-2,4,6-triyl)tris(Ncyanocyanamide). The isolated yield was $79 \%(0.22 \mathrm{~g}, 0.79$ $\mathrm{mmol})$. IR $\left(\nu / \mathrm{cm}^{-1}\right)$ : $3172 \mathrm{w}, 2186 \mathrm{~s}\left[\left(\mathrm{~N}-(\mathrm{CN})_{2}\right)\right], 1616$ $\mathrm{s}$ [triazine ring], $1391 \mathrm{~s}$. Elem. Anal. Calcd. \% (Found \%) for $\mathrm{C}_{9} \mathrm{~N}_{12}$ : C, 39.14 (39.26); N, 60.86 (60.75). LCMS: $\mathrm{M}^{+}$ 277. HRMS: Calculated mass (observed mass), 276.0369 (277.1790). ${ }^{13} \mathrm{C}$ NMR (solid): $\delta 119.34,162.13$.

\subsection{Synthesis of 2,4,6-tris[bis(1H-tetrazol-5-yl) amino]-1,3,5-triazine (2)}

An aqueous solution [ $40 \mathrm{~mL}$ of deionized water] of 1 ( $0.91 \mathrm{~g}$, $3.3 \mathrm{mmol})$, sodium azide ( $1.43 \mathrm{~g}, 22 \mathrm{mmol})$ and zinc bromide $\left(4.50 \mathrm{~g}, 20 \mathrm{mmol}\right.$ ) was refluxed (at $140^{\circ} \mathrm{C}$ of oil bath) for 24 $\mathrm{h}$ with vigorous stirring. After cooling to room temperature, the reaction mixture was cooled further by adding crushed ice. Then, the stirring was continued for another $30 \mathrm{~min}$ and the reaction mixture was filtered and washed with cold water. After that, the resultant product was air-dried to obtain a white, powdered sample (2). The isolated yield was $73 \%(1.29 \mathrm{~g}$, $2.4 \mathrm{mmol})$. IR $\left(\nu / \mathrm{cm}^{-1}\right): 3413 \mathrm{~s}, 3095 \mathrm{~m}, 1610 \mathrm{~s}, 1468 \mathrm{~s}$, 1084 s, 794 s. Elem. Anal. Calcd. \% (Found \%) for $\mathrm{C}_{9} \mathrm{H}_{6} \mathrm{~N}_{30}$ : C, 20.23 (20.15); H, 1.13 (1.21); N, 78.64 (78.52). LCMS: $\mathrm{M}^{+}$535. HRMS: Calculated mass (observed mass), 534.1392 (534.2941). ${ }^{1} \mathrm{H}$ NMR (DMSO-d6): $\delta 7.308$ (6H, s). ${ }^{13} \mathrm{C}$ NMR (DMSO-d6): $\delta$ 165.0, 154.8.

\section{Results and Discussion}

Cyanuric chloride was used as the starting material for the synthesis of compounds $\mathbf{1}$ and $\mathbf{2}$. The synthesis of $\mathbf{1}$ was carried out by the reaction of cyanuric chloride with sodium dicyanamide (1:3 ratio) in acetone-water (80:20 ratio) mixture at 50 to $60^{\circ} \mathrm{C}$ for one hour (Scheme 1). The compound 1 was obtained in $79 \%$ yield as a white powder that was insoluble in water and common organic solvents. The mid-IR spectrum of the compound 1 showed a band at $2190 \mathrm{~cm}^{-1}$ corresponding to the stretching vibration of the $\mathrm{CN}$ groups. The peaks at $1630-1650,1380$ and $1460 \mathrm{~cm}^{-1}$ were related to the triazine ring stretching frequencies (Figure S1 in Supplementary Information). Further, the structure of $\mathbf{1}$ was confirmed by solid-state ${ }^{13} \mathrm{C}$ NMR, elemental analysis, LCMS, and HRMS. The dried, powdered $\mathbf{1}$ was used in the reaction to synthesize compound $\mathbf{2}$.

The attractive syntheses of novel energetic products include a broad range of neutral molecules and salts of tetrazole due to their high nitrogen content $(\sim 80 \%)$, the high positive heat of formation $(320 \mathrm{~kJ} / \mathrm{mol})$, low sensitivity towards impact and excellent thermal stability that results from the aromatic ring system. The introduction of multiple heterocyclic rings within a single cation has been proved to possess promising energetic properties. ${ }^{9}$ The reaction of 1 with sodium azide and zinc bromide in deionized water at the reflux (oil bath temperature of $140^{\circ} \mathrm{C}$ ) for $24 \mathrm{~h}$ resulted in the formation of compound 2 (Scheme 1). ${ }^{14,15}$ The crude product 2 was obtained as a precipitate, which was isolated by filtration. The compound 2 was characterized using FTIR and NMR spectral data.

In the FTIR spectrum, the stretching vibration of dicyanamide ion disappeared, and new peaks related to tetrazole was observed at $795,1081,1460,1617$, 3103,3277 , and $3423 \mathrm{~cm}^{-1}$ (Figure S2 in Supplementary Information). The ${ }^{13} \mathrm{C}$ NMR spectrum (Figure S3 in SI) showed two peaks at $156.34 \mathrm{ppm}$ and $165.82 \mathrm{ppm}$ corresponding to tetrazole and triazine rings; in ${ }^{1} \mathrm{H}$ NMR signal (Figure S4 in SI) at the $7.31 \mathrm{ppm}$ indicates the presence of $\mathrm{N}-\mathrm{H}$ of tetrazole. Also, the product was characterized by the elemental analysis, LCMS, and HRMS. Though many compounds were known with tetrazole groups, this is the first example of a compound having 


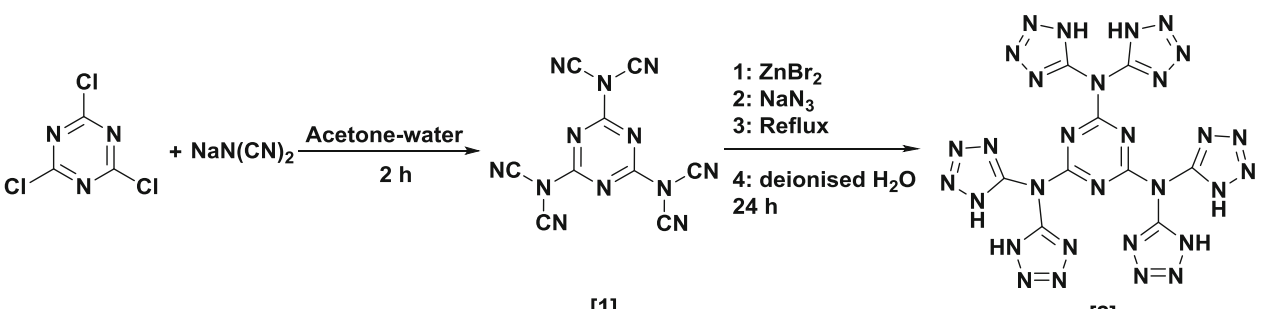

[1]

[2]

Scheme 1. Synthesis of compounds $\mathbf{1}$ and $\mathbf{2}$.

Table 1. Energetic properties of compounds $\mathbf{1}$ and $\mathbf{2 .}$

\begin{tabular}{lccllllllc}
\hline \multirow{2}{*}{ Compound } & MF & NC $\%$ & OB $\%$ & D & HOF & VOD & DP & IS & FS \\
\hline $\mathbf{1}$ & $\mathrm{C}_{9} \mathrm{~N}_{12}$ & 61 & -104 & 1.62 & 1314.4 & 6.18 & 15.87 & $>50$ & $>360$ \\
$\mathbf{2}$ & $\mathrm{C}_{9} \mathrm{H}_{6} \mathrm{~N}_{30}$ & 79 & -63 & 1.88 & 1833.0 & 7.43 & 25.11 & $>50$ & $>360$ \\
RDX & $\mathrm{C}_{3} \mathrm{H}_{6} \mathrm{~N}_{6} \mathrm{O}_{6}$ & 38 & -21.6 & 1.80 & 83.82 & 8.75 & 33.8 & 7.4 & $>120$ \\
$\mathrm{HMX}$ & $\mathrm{C}_{4} \mathrm{H}_{8} \mathrm{~N}_{8} \mathrm{O}_{8}$ & 38 & -21.6 & 1.90 & 104.77 & 9.10 & 39.0 & 7.4 & $>112$ \\
TNT & $C_{7} \mathrm{H}_{5} \mathrm{~N}_{3} \mathrm{O}_{6}$ & 19 & -74.0 & 1.65 & -54.39 & 6.90 & 19.0 & 15 & $>353$ \\
\hline
\end{tabular}

MF: Molecular Formula, NC: Nitrogen Content. OB: Oxygen Balance, D: Density in $\left(\mathrm{g} \mathrm{cm}^{-3}\right)$, HOF: Heat of formation in the solid phase $\left(\mathrm{kJ} \mathrm{mol}^{-1}\right)$, VOD: Velocity of Detonation $\left(\mathrm{km} \mathrm{s}^{-1}\right)$, DP: Detonation Pressure (Gpa), IS: Impact Sensitivity (J), FS: Friction Sensitivity (N). (Data for RDX, HMX and TNT are taken from the literature). ${ }^{22 c, d, e}$

six tetrazole rings in a single molecule constructed on triazine ring.

All the DFT calculations were performed using Gaussian 09 program package. ${ }^{16}$ The geometries of $\mathbf{1}$ and $\mathbf{2}$ were optimized at the B3PW91 level with 6-31G(d,p) basis set. The computational methods used were similar to our previous reports. ${ }^{17}$ The gas phase HOF was predicted using isodesmic reactions while Politzer approach ${ }^{18}$ was used to compute heat of sublimation. The resultant heat of sublimation was deducted from the gas phase HOF to obtain solid phase HOF of $\mathbf{1}$ and 2. The solid phase HOFs of $\mathbf{1}$ and $\mathbf{2}$ were 1314 and $1833 \mathrm{~kJ} \mathrm{~mol}^{-1}$, respectively. These high positive $\mathrm{HOFs}$ attributed to more number of $\mathrm{C}-\mathrm{N}$ and $\mathrm{N}-\mathrm{N}$ bonds present in their structures. The compound 2 could be used as enthalpy enhancer due to the high HOF. The molecular surface properties were calculated via the Multiwfn program ${ }^{19}$ and used to predict the densities. ${ }^{20}$ Compound 2 showed superior density $\left(1.88 \mathrm{~g} \mathrm{~cm}^{-3}\right)$ to $\operatorname{RDX}\left(1.80 \mathrm{~g} \mathrm{~cm}^{-3}\right)$ while 1 showed density of $1.62 \mathrm{~g}$ $\mathrm{cm}^{-3}$. The detonation velocity (VOD in $\mathrm{km} \mathrm{s}^{-1}$ ) and detonation pressure (DP in GPa) were computed using Kamlet-Jacobs empirical equations. ${ }^{21}$ The VOD (7.43 $\mathrm{km} \mathrm{s}^{-1}$ ) and DP (25.11 GPa) of $\mathbf{2}$ are found to be higher than those of TNT $\left(\mathrm{VOD}=6.90 \mathrm{~km} \mathrm{~s}^{-1}, \mathrm{DP}=19.0 \mathrm{GPa}\right)$.

The performance and safety characteristics of molecules need to be evaluated for safe handling of compounds. Impact and friction sensitivity are more important characteristics in the manufacturing context. Therefore, the impact and friction sensitivities of the compound 2 were measured using the standard BAM fall hammer, and BAM friction techniques (Table 1). ${ }^{22}$ The impact sensitivity of compounds $\mathbf{1}$ and $\mathbf{2}$ was above $50 \mathrm{~J}$ while the friction sensitivity of compounds $\mathbf{1}$ and 2 was $>360 \mathrm{~N}$. This sensitivity data revealed that the compound $\mathbf{2}$ was an insensitive material and much less sensitive than TNT, RDX and HMX. Thermal stability is an essential parameter for any explosive in processing and storage, which need explosives that can withstand high temperatures. For common usage, a component explosive must be stable above $200^{\circ} \mathrm{C}$. As observed in the thermogravimetric analyses (Figures S5 and S6 in Supplementary Information), the compounds $\mathbf{1}$ and $\mathbf{2}$ with a decomposition onset of $300^{\circ} \mathrm{C}$ certainly exceed this requirement.

\section{Conclusions}

In summary, the molecule, 2,4,6-tris[bis $(1 \mathrm{H}$-tetrazol5-yl)amino]-1,3,5-triazine (2) having 79\% nitrogen content was synthesized from cyanuric chloride in a two-step process with a $58 \%$ yield. Its structure was confirmed using spectroscopic methods, including FTIR and NMR spectroscopy $\left({ }^{1} \mathrm{H}\right.$ and $\left.{ }^{13} \mathrm{C}\right)$, elemental analysis, and HRMS. The high positive HOF values of $\mathbf{1}$ and 2 showed that these compounds could be potential enthalpy enhancers in energetic formulations. The high nitrogen content associated with high positive heat of formation of the compound $\mathbf{2}$ underlines the structural importance of six tetrazole groups in a molecule. 
According to the data, these compounds are possible energetic materials; however, an extensive performance evaluation needs to be carried out to prove their usage in the Science and Technology of Propellants.

\section{Supplementary Information (SI)}

Electronic Supplementary Information (ESI) available are FTIR, NMR spectra, TGDTA curves and computational details of compounds [1] and [2]. Supplementary Information is available at www.ias.ac.in/chemsci.

\section{Acknowledgements}

The authors gratefully acknowledge the funding from DRDO, India in the form of a research grant to ACRHEM. The authors also thank School of Chemistry, the University of Hyderabad for infrastructure. V.D.G. thanks the DST-SERB for a research grant (Young Scientists, No. SB/FT/CS-110/2014).

\section{References}

1. (a) Agrawal J P and Hodgson R D 2007 In Organic Chemistry of Explosives (Chichester, UK: John Wiley) p. 293; (b) Akhavan J 1998 In The Chemistry of Explosives (Cambridge: The Royal Society of Chemistry) p.170; (c) Klapötke T M 2007 In High Energy materials: Structure and Bonding (Berlin: Springer) p.125

2. (a) Badgujar D M, Talawar M B, Asthana S N and Mahulikar P P 2008 Advances in science and technology of modern energetic materials: An overview $J$. Hazard. Mater. 151 289; (b) Nair U R, Asthana S N, Rao A S and Gandhe B R 2010 Advances in High Energy Materials Def. Sci. J. 60 137; (c) Talawar M B, Sivabalan R, Mukundan T, Muthurajan H, Sikder A K, Gandhe B R and Rao A S 2009 Environmentally compatible next generation green energetic materials (GEMs) J. Hazard. Mater. 161 589; (d) Hanumantha Rao $\mathrm{M}$ and Muralidharan K 2016 Closo-Dodecaborate $\left(\mathrm{B}_{12}\right.$ $\left.\mathrm{H}_{12}\right)^{2-}$ salts with nitrogen based cations and their energetic properties Polyhedron 115 105; (e) Guo Y, Gao H, Twamley B and Shreeve J M 2007 Energetic Nitrogen Rich Salts of $N, N$-bis[1(2)H-Tetrazol-5-yl]amine $A d v$. Mater. 19 2884; (f) Guo Y, Tao G -H, Zeng Z, Gao H, Parrish D A and Shreeve J M 2010 Energetic Salts Based on Monoanions of $N, N$-Bis (1H-tetrazol-5-yl)amine and 5,5'-Bis(tetrazole) Chem. Eur. J. 163753

3. (a) Sikder A K and Sikder N 2004 A review of advanced high performance, insensitive and thermally stable energetic materials emerging for military and space applications J. Hazard. Mater. 112 1; (b) Wang R, Xu H, Guo Y, Sa R and Shreeve J M 2010 Bis[3-(5-nitroimino1,2,4-triazolate)]-Based Energetic Salts: Synthesis and Promising Properties of a New Family of High-Density Insensitive Materials J. Am. Chem. Soc. 13211904

4. (a) Gao H and Shreeve J M 2011 Azole-Based Energetic Salts Chem. Rev. 111 7377; (b) Wang R, Guo Y, Zeng
Z and Shreeve J M 2009 Nitrogen-rich nitroguanidylfunctionalized tetrazolate energetic salts Chem. Commun. 2697; (c) Wang R, Guo Y, Zeng Z, Twamley B and Shreeve J M 2009 Furazan-Functionalized TetrazolateBased Salts: A New Family of Insensitive Energetic Materials Chem. Eur. J. 15 2625; (d) Tao J and Wang X 2017 Crystal structure and morphology of $\beta$-HMX in acetone: A molecular dynamics simulation and experimental study J. Chem. Sci. 129 495; (e) Li M, Wu F $\mathrm{M}$ and Xu H 2017 Molecular design and performance prediction of poly-dinitroamino pyrrole compounds as energetic materials J. Chem. Sci. 12957

5. (a) Singh R P, Verma R D, Meshri D T and Shreeve J M 2006 Energetic Nitrogen-Rich Salts and Ionic Liquids Angew. Chem. Int. Ed. 45 3584; (b) Tao G-H, Guo Y, Parrish D A and Shreeve J M 2010 Energetic 1,5-diamino-4H-tetrazolium nitro-substituted azolates $J$. Mater. Chem. 202999

6. (a) Chavez D E and Hiskey M A 1999 1,2,4,5-tetrazine based energetic materials J. Energetic Mater. 17 357; (b) Tao G-H, Guo Y, Joo Y -H, Twamley B and Shreeve J M 2008 Energetic nitrogen-rich salts and ionic liquids: 5-aminotetrazole (AT) as a weak acid J. Mater. Chem. 185524

7. (a) Dippold A A and Klapötke T M 2013 Synthesis and Characterization of 5-(1,2,4-Triazol-3-yl)tetrazoles with Various Energetic Functionalities Chem. Asian J. 8 1463; (b) Thottempudi V, Zhang J, He C and Shreeve J M 2014 Azo substituted 1,2,4-oxadiazoles as insensitive energetic materials RSC Adv. 450361

8. Hafner K, Klapötke T M, Schmid P C and Stierstorfer J 2015 Synthesis and Characterization of the Asymmetric 1,2-Dihydroxy-5,5'-bitetrazole and Selected NitrogenRich Derivatives Eur. J. Inorg. Chem. 172794

9. Srinivas D, Ghule V D, Muralidharan K and B Jenkins H D 2013 Tetraanionic Nitrogen-Rich Tetrazole-Based Energetic Salts Chem. Asian J. 81023

10. (a) Joo Y H and Shreeve J M 2008 1-Substituted 5Aminotetrazoles: Syntheses from $\mathrm{CNN}_{3}$ with Primary Amines Org. Lett. 10 4665; (b) Steinhauser G, Giester G, Wagner C, Weinberger P, Zachhuber B, Ramer G, Villa M and Lendl B 2012 Nitrogen-rich Compounds of the Actinoids: Dioxouranium(VI) 5,5/- Azobis[tetrazolide] Pentahydrate and Its Unusually Small Uranyl Angle Inorg. Chem. 51 6739; (c) Klapötke T M and Sabate C M 2009 New energetic compounds based on the nitrogenrich 5,50 -azotetrazolate anion $\left(\left[\mathrm{C}_{2} \mathrm{~N}_{10}\right]^{2-}\right)$ New J. Chem. 331605

11. Pagoria P F, Lee G S, Mitchell A R and Scmidt R D 2002 A review of energetic materials synthesis Thermochim. Acta 384187

12. Fried L E, Manaa M R, Pagoria P F and Simpson R L 2001 Design and Synthesis of Energetic Materials Anпи. Rev. Mater. Res. 31291

13. Turker L, Atalar T, Gumus S and Camur Y 2009 A DFT study on nitrotriazines J. Hazard. Mater. 167440

14. Demko Z P and Sharpless K B 2001 Preparation of 5Substituted 1H-Tetrazoles from Nitriles in Water J. Org. Chem. 667945

15. Chafin A, Irvin D J, Mason M H and Mason S L 2008 Synthesis of multifunctional hydroxyethyl tetrazoles Tetrahedron Lett. 493823 
16. Frisch M J 2013 et al.,Gaussian 09, Revision A.1 (Wallingford CT, Gaussian Inc.)

17. (a) Ghule V D 2012 Computational Studies on Energetic Properties of Trinitro Substituted Imidazole-Triazole and Pyrazole-Triazole Derivatives J. Phys. Chem. A 116 9391; (b) Deswal S, Ghule V D, Tittal R K and Radhakrishnan S 2015 Quantum-chemical design of tetrazolo[1,5-b][1,2,4,5]tetrazine based nitrogen-rich energetic materials Comput. Theor. Chem. 1054 55; (c) Srinivas D, Ghule V D and Muralidharan K 2014 Synthesis of nitrogen-rich imidazole, 1,2,4-triazole and tetrazole-based compounds $R S C A d v .47041$; (d) Srinivas D, Ghule V D and Muralidharan K 2014 Energetic salts prepared from phenolate derivatives New J. Chem. 38 3699; (e) Hanumantha Rao M and Muralidharan K 2013 Syntheses, characterization and energetic properties of closo-(B12H12) ${ }^{2-}$ salts of imidazolium derivatives Dalton. Trans. 428854

18. Politzer P, Ma Y, Lane P and Concha M C 2005 Computational Prediction of Standard Gas, Liquid, and Solid-Phase Heats of Formation and Heats of Vaporization and Sublimation Int. J. Quant. Chem. 105 341

19. Lu T and Chen F 2012 Multiwfn: A multifunctional wavefunction analyzer J. Comput. Chem. 33580
20. Politzer P, Martinez J, Murray J S, Concha M C and ToroLabbé A 2009 An electrostatic interaction correction for improved crystal density prediction Mol. Phys. 1072095

21. Kamlet M J and Jacobs S J 1968 Chemistry of Detonations. I. A Simple Method for Calculating Detonation Properties of C-H-N-O Explosives J. Chem. Phys. 48 23

22. (a) http://www.bam.de; (b) About $20 \mathrm{mg}$ of compound 2 was subjected to a drop-hammer test using a 5 or $10 \mathrm{~kg}$ dropping weight. A range in impact sensitivities according to the UN Recommendations (insensitive $>40 \mathrm{~J}$; less sensitive $\geq 35 \mathrm{~J}$; sensitive $\geq 4 \mathrm{~J}$; very sensitive $\leq 3 \mathrm{~J}$ ). Friction, Insensitive $>360 \mathrm{~N}$, less sensitive $=360 \mathrm{~N}$; sensitive $>360 \mathrm{~N}<80 \mathrm{~N}$; very sensitive $\leq 80 \mathrm{~N}$; extremely sensitive $\leq 10 \mathrm{~N}$; according to the United nations recommendations on the transport of dangerous goods; (c) Mayer R, Köhler J and Homburg A 2002 Explosives 5th ed. (Weinheim: Wiley-VcH) (d) Tang Y, Yang H, Ju X, Huang H, Lua C and Cheng G 2014 A novel $\mathrm{N}-\mathrm{N}$ bond cleavage in 1,5- diaminotetrazole: synthesis and characterization of 5-picrylamino-1,2,3,4-tetrazole (PAT) J. Mater. Chem. A 24127; (e) Fischer N, Fischer D, Klapötke T M, Piercey D G and Stierstorfer J 2012 Pushing the limits of energetic materials - the synthesis and characterization of dihydroxylammonium 5,50 -bistetrazole-1,10 -diolate J. Mater. Chem. 2220418 